\title{
XI \\ The Interplay of Risk Factors
}

The risk factors that might have contributed to the eighteenth-century English emergence of recognizable angina pectoris and its subsequent increase in frequency have hitherto been considered individually. Age, dietary factors, obesity, limited physical activity, use of tobacco, stress and genetic factors have all been examined in isolation. Of necessity, epidemiologists have to follow this course in order to establish whether or not any one suspected risk factor is or is not associated with an increase in the incidence of coronary heart disease. Multivariate analysis is therefore always used in order to eliminate the impact of any factor not under immediate study.

In the real world one or more, or even all of the traditional risk factors often coexist, either in any one individual or in clearly defined groups of people, and they do so with a frequency greater than would be expected by chance alone. In some cases an individual may have adopted a life pattern that involved more than one risk factor. Often, for example, a diet is unhealthy in several respects. High fat and carbohydrate consumption tend to go together and both are associated with low fibre intake. An inverse relationship tends to govern consumption of fish on the one hand and animal foods including fats on the other. Coffee is frequently taken with both cream and sugar, and in the eighteenth century the patrons of coffee houses suffered secondary exposure to tobacco smoke for long periods and in high concentrations. A high fat diet is often associated with heavy smoking. Obesity is linked with low physical activity and low physical activity is conducive to obesity.

Some risk factors, even if adopted singly by an individual, lead inexorably to others. Excessive consumption of fat, and of saturated fats in particular, is associated with both systolic and diastolic hypertension, ${ }^{1}$ as are high coffee, salt and energy intake, and diets low in fibre. Sucrose (cane sugar) elevates the total serum cholesterol level for up to eight hours when it is taken in conjunction with a high fat diet. ${ }^{2}$ When compared to people on a high fibre diet, those with relatively low fibre intake tend to have not only higher blood pressures, but also elevated serum total cholesterol and lower HDL levels, i.e. they show adverse trends in their lipid profiles. ${ }^{3}$ The latter are also associated with smoking and mental stress, and these two are often associated. An extreme example of risk factors being combined is seen in the patient with syndrome $\mathrm{X}$, a combination of truncal obesity, hypertension, elevated serum

\footnotetext{
' Pirjo Pietinen et al., 'Dietary fat and blood pressure-a review', Eur Heart J, 1987, 8: Suppl B9-17, p. 11.

${ }^{2}$ Kevin I Grant, Martelle P Marais and Muhammad A Dhansay, 'Sucrose in a lipid-rich meal amplifies the postprandial excursion of serum and lipoprotein triglyceride and cholesterol concentrations by decreasing triglyceride clearance', Am J Clin Nutr, 1994, 59: 853-60, p. 856.

${ }^{3}$ Angela Wright, P G Burstyn and M J Gibney, 'Dietary fibre and blood pressure', Br Med J, 1979, ii: $1541-3$, p. 1542 .
} 


\section{The Interplay of Risk Factors}

triglycerides, raised blood sugar, and insulin resistance. ${ }^{4}$ Increasing age impacts unfavourably on all other risk factors.

There is now good evidence to show that when more than one risk factor is present, either in an individual or in a population, the impact on the likelihood of coronary heart disease developing is often characterized not only by an additive, but also by a multiplicative effect. John Muir and David Mant summarized the MRFIT findings concerning the impact of three risk factors on male coronary heart disease rates. In the absence of any of these risk factors as defined by the authors, the six-year "basic" mortality rate was $3.0 / 1000$. By themselves, smoking, serum total cholesterol elevation and a high diastolic blood pressure were associated with increases above the "basic" in six-year death rates of 5.9, 6.9 and 4.7 per thousand respectively. On a simple additive basis, the three factors together would have accounted for an excess cardiac mortality rate of 17.5 per thousand. In fact, the subgroup in which all three were present suffered an excess six-year death rate per thousand of 30.5. In a small population, these conclusions might have been invalidated by failure of the investigators to stratify by the actual number of cigarettes smoked or the degree of elevation of the serum cholesterol and diastolic blood pressure levels. However, this study involved a total of about one-third of a million men, so that any such variations in risk factor "intensity" would have averaged out. The increase in relative risk produced by smoking was shown clearly to be greater when either the diastolic blood pressure, the total serum cholesterol or both were in the higher category. Figure XI.1 shows that the effects of elevated serum triglycerides on CHD incidence are greatly magnified when the total serum cholesterol is also elevated. All of these findings indicate that there is a multiplicative effect when two or more traditional risk factors co-exist in any one patient. ${ }^{5}$

There is increasing evidence to suggest that blood levels of some of the more recently recognized CHD risk factors, although genetically determined in varying degree, are of more serious consequence when also associated with presence of traditional factors. Those newly recognized include elevation of serum lipoprotein (a) and homocysteine levels and a variety of plasma clotting factors, notably fibrinogen and factor VII. A five-year prospective study of 2,156 men free of clinical CHD and aged forty-seven to sixty-four years at entry showed that the association of an elevated $\mathrm{Lp}$ (a) level with a high risk of subsequent CHD is significantly increased when the serum LDL cholesterol is in the highest tertile (RR 2.15, CL 1.34-3.44). ${ }^{6}$ Serum concentrations of the amino-acid homocysteine rise with age and are higher in men and in smokers. ${ }^{7}$ Platelet aggregation, which initiates blood

\footnotetext{
${ }^{4}$ Syndrome X, American Heart Association internet posting, http://www.americanheart.org/.

'John Muir and David Mant, 'Multiple risk', in Martin Lawrence et al. (eds), Prevention of cardiovascular disease: an evidence-based approach, Oxford University Press, 1996, p. 187; G Assmann and J H Schulte, 'Relation of high-density lipoprotein cholesterol and triglycerides to incidence of atherosclerotic coronary artery disease (the PROCAM experience)', Am J Cardiol, 1992, 70: 733-7, p. 736.

${ }^{6}$ Bernard Cantin et al., 'Is lipoprotein (a) an independent risk factor for ischemic heart disease in men? The Quebec cardiovascular study', J Am Coll Cardiol, 1998, 31: 519-25, pp. 521-2.

${ }^{7}$ Ottar Nygård et al., 'Total plasma homocysteine and cardiovascular risk profile. The Hordaland homocysteine study', JAMA, 1995, 274: 1526-33, pp. 1526-7.
} 
CAD cases $/ 1,000$ in 6 years

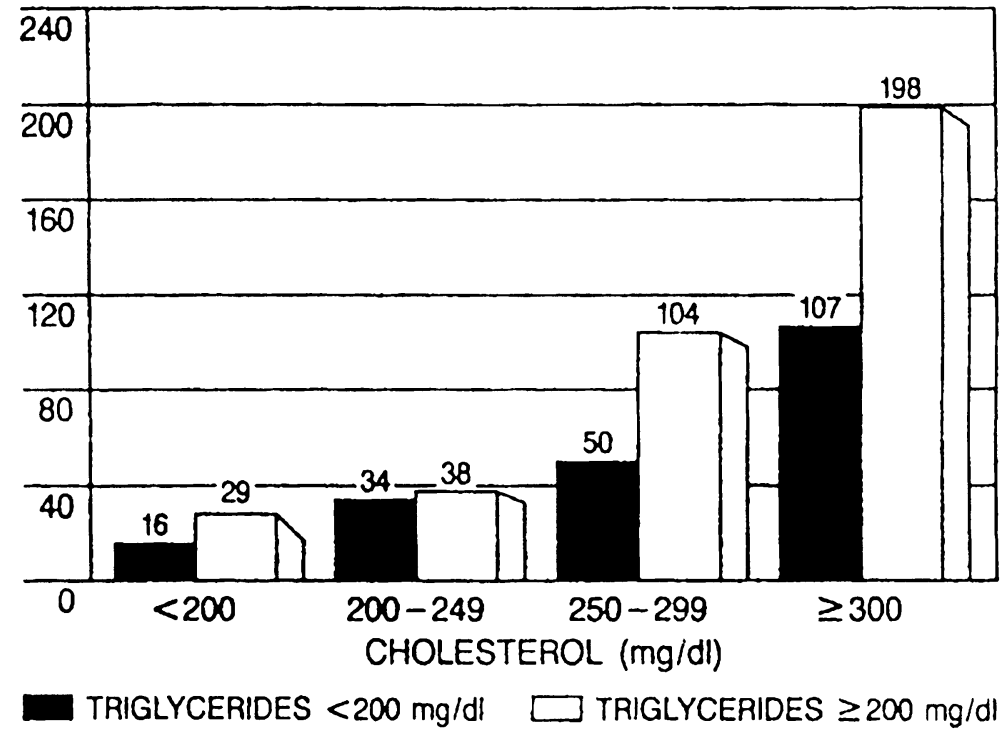

Figure XI.1: Incidence of CHD/1000 subjects over a 6-year follow-up in relation to triglyceride and cholesterol levels. Reproduced from G Assmann and H Schulte, 'Relation of high-density lipoprotein cholesterol and triglycerides to incidence of atherosclerotic coronary artery disease (the PROCAM experience)', Am J Cardiol, 1992, 70: 733-7, p. 736. (With permission from Excerpta Medica Inc.)

coagulation, is enhanced when the lipid profile is abnormal and clot formation is promoted as a consequence. ${ }^{8}$ Clotting factor VII activity correlates positively with both total and serum HDL cholesterol levels. ${ }^{9}$ In men the plasma levels of the clot precursor fibrinogen are positively correlated with physical inactivity, smoking and with total serum cholesterol, triglyceride and LDL levels. ${ }^{10}$ In the PROCAM study it was found that the impact of plasma fibrinogen levels on the combined incidence of sudden cardiac death and myocardial infarction was more than doubled when the serum cholesterol level was in the highest tertile. " The Whitehall health study of British civil servants showed plasma fibrinogen levels to be significantly higher

${ }^{8}$ Angelina C A Carvalho, Robert W Colman and Robert S Lees, Platelet function in hyperlipoproteinemia', $N$ Engl J Med, 1974, 290: 434 8, p. 435.

${ }^{9}$ Carol J Hoffman, Robin H Miller and Mae B Hultin, 'Correlation of factor VII activity and antigen with cholesterol and triglycerides in healthy young adults', Arterioscler Thromh, 1992, 12: 267-70, p. 269.

${ }^{10}$ Edzard Ernst and Karl L Resch, 'Fibrinogen as a cardiovascular risk factor: a meta-analysis and review of the literature', Ann Intern Med, 1993, 118: 956-63, p. 959.

"Jürgen Heinrich et al., 'Fibrinogen and factor VII in the prediction of coronary risk. Results from the PROCAM study in healthy men", Arterioscler Thromb, 1994, 14: 54 9, p. 56. 
among smokers when compared to non-smokers. ${ }^{12}$ Similar differences were also observed when the job-stressed personnel in the lowest employment categories were compared with the relatively unstressed members of the highest civil service grades. The plasma fibrinogen levels of the former were the higher and the differences significant. ${ }^{13}$

During the eighteenth century there was, as pointed out in earlier chapters, an increase in both the relative and the absolute numbers of the English middle and upper classes and a growing tendency for them to live to a greater age than the general population. These relatively privileged members of society embraced a pattern of living that included low intake of fibre, but consumption of increasing amounts of animal fats, coffee, sugar and salt and a tendency to become obese in later life. Tobacco products were being used more extensively. Upward mobility with adoption of affluent living styles was associated with a decrease in physical activity as servants became affordable. The middle classes probably walked and rode on horseback less during the course of the eighteenth century as roads became better and horse-drawn vehicles improved in availability, speed, comfort and efficiency. Some causes of the mental stresses to which the commercial classes were uniquely subject appear to have increased during the Georgian era. In England, therefore, the multiplicative effects of the various risk factors in combination could well have accentuated the contribution of the individual elements to the late eighteenth-century development of coronary heart disease with its overt clinical manifestations, notably angina of effort.

\footnotetext{
${ }^{12}$ Eric Brunner et al., 'Childhood social circumstances and psychosocial behavioural factors as determinants of plasma fibrinogen', Lancet, 1996, 347: 1008-13, p. 1011.

${ }^{13} \mathrm{H}$ L Markowe et al., 'Fibrinogen: a possible link between social class and coronary heart disease', Br Med J, 1985, 291: 1312-14, p. 1313.
} 